\title{
Study on the Fabrication Process of Polarization Maintaining Photonic Crystal Fibers and Their Optical Properties
}

\author{
Tai Yong Cho, ${ }^{1,2}$, Gil Hwan Kim, Kwanll Lee, and Sang Bae Lee* \\ ${ }^{1}$ Photonics Research Laboratory, Division of Intelligent System Research, Korea Institute of Science \\ and Technology (KIST), Seoul 130-791, Korea \\ Je-Myung Jeong \\ ${ }^{2}$ Optical Communication Laboratory, Division of Electrical and Computer Engineering, \\ Hanyang University, Seoul 133-791, Korea
}

(Received January 11, 2008 : revised February 13, 2008)

\begin{abstract}
In this paper, we describe the fabrication process and the characteristics of polarization maintaining photonic crystal fibers (PM-PCFs). The PM-PCF is fabricated by stack-and-draw method, i.e., stacking silica capillary tubes (making a PM-PCF preform) and drawing to optical fiber. Firstly, a PM-PCF preform is formed by stacking two kinds of capillary tubes around a solid silica rod and jacketing these stacked tubes with an outer silica tube (out-jacket tube). Later, the desired preform is drawn to a fiber in a high temperature drawing tower. We also compare the polarization properties such as polarization dependent loss, birefringence, and differential group delay of the fabricated PM-PCF with those of the conventional PANDA PM fiber.
\end{abstract}

OCIS codes : 060.0060, 060.2400

\section{INTRODUCTION}

In recent years, photonic crystal fiber (PCF) has attracted significant interest because of its unusual properties and new applications in telecommunications and novel photonic devices. PCF is characterized by a series of regularly arranged air holes running along the fiber length and a defect region in its center. Generally, PCF can be divided into two categories according to the light guiding mechanism; index-guiding photonic crystal fiber and photonic bandgap fiber (PBGF). The index-guiding PCF guides light by modified total internal reflection (M-TIR) between a solid core (usually undoped silica) and a cladding region having multiple air holes. On the other hand, the PBGF uses photonic bandgap effect to guide light in a hollow (air) core. In a PBGF, the core is created by introducing a defect (i.e. air hole) in the PBG structure, thereby creating an area where the light can propagate. As the light can only propagate at the defect region, a low index guiding core is possible. Light can be freely controlled and transformed in these fibers by the air-hole structure. Therefore, PCFs have many unique properties such as endlessly single-mode operation over a wide wavelength range [1], large mode field diameter [2], dispersion control by air hole structure [3], high birefringence [4], nonlinear effect [5], low bending loss [6] and supercontinuum generation compared to conventional optical fibers.

PCF can be also made highly birefringent by introducing anisotropy into the fiber structure [4]. Polarization maintaining (PM) PCF has different air hole diameters along two orthogonal axes near the core region, which induce a high effective index difference between the two orthogonal polarization modes. It has been shown that its birefringence is of the order of $10^{-3}$, three times larger than that of conventional PANDA [7] or bow-tie fibers. [8]

In this paper, we report the fabrication process of PM-PCF and the optical properties of the fabricated PM-PCF; we make two kinds of silica capillaries and core rod by drawing a silica tube and silica rod, respectively. These capillaries are stacked around the silica core rod by hands into a hexagonal structure and inserted into the jacketing tube (out-jacket tube). Note that two capillaries with larger inner diameter are located at both sides of the silica core rod. This preform is moved into the high temperature furnace of a drawing tower and then fused together and drawn down 
to a size of $5 \sim 6 \mathrm{~mm}$ cane. After an additional sleeve tube is added, it is drawn down to final PM-PCF in a second drawing stage. Here, we use two drawing processes to maintain uniform air hole structure. The birefringence and loss of the fabricated PM-PCF were measured to be $1 \times 10^{-3}$ and $0.04 \mathrm{~dB} / \mathrm{m}$, respectively.

\section{DESIGN OF PM-PCF}

In order to induce large birefringence into the PM-PCF, we incorporated two larger air holes at both sides of the core. The birefringence arises from the effective refractive index difference between the $x$ (fast axis) and $y$ (slow axis) axis of polarization modes.

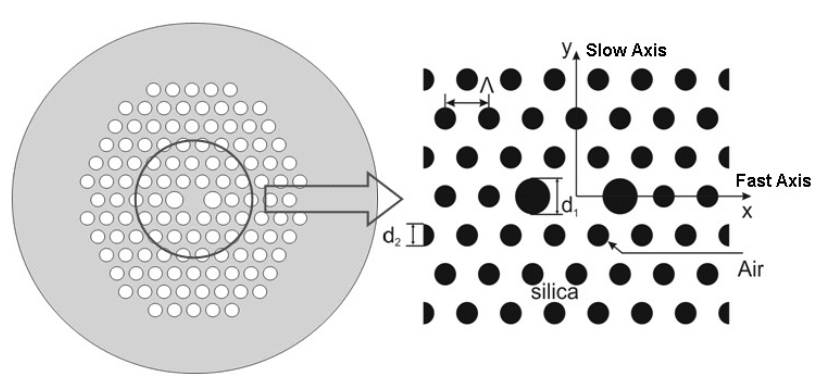

(a)

(b)

FIG. 1. Desired cross section (a) and the structural model (b) of PM-PCF.
Therefore, each diameter of the large $\left(\mathrm{d}_{1}\right)$ and small air hole $\left(\mathrm{d}_{2}\right)$ gives the birefringent characteristics of PM$\mathrm{PCF}$ and the ratio between the diameter of small air hole $\left(\mathrm{d}_{2}\right)$ and large air hole $\left(\mathrm{d}_{1}\right)\left(\mathrm{d}_{2} / \mathrm{d}_{1}\right)$ determines the modal birefringence of the fiber. When $d_{2} / d_{1}=1$, it is identical to large-mode-area (LMA) PCF and there is no modal birefringence. The modal birefringence increases as $d_{2} / d_{1}$ decreases. This is due to the fact that the six-fold symmetry is destroyed with a decrease in the $\mathrm{d}_{2} / \mathrm{d}_{1}$ ratio and the effective refractive index difference of two orthogonal polarization modes changes. So, we targeted $\mathrm{d}_{2} / \mathrm{d}_{1}$ as 0.5 in order to obtain high birefringence.

\section{FABRICATION PROCESS OF PM-PCF}

The fabrication process of PM-PCF is a little different from that of the conventional PM fiber. Fig. 2 shows the fabrication process of PM-PCF using capillary stacking method.

The first process is to draw a $1 \mathrm{~mm}$ silica rod for core. The second process is to collapse two silica tubes in order to get the two kinds of silica tubes with different inner and outer diameters. This collapsing process is carried out in the lathe of MCVD (Modified Chemical Vapor Deposition). Later, these two collapsed silica tubes (Collapsed Tube (1) and Collapsed Tube (2), respectively) are drawn to $1 \mathrm{~mm}$ capillaries which have different inner diameter but the same outer diameter

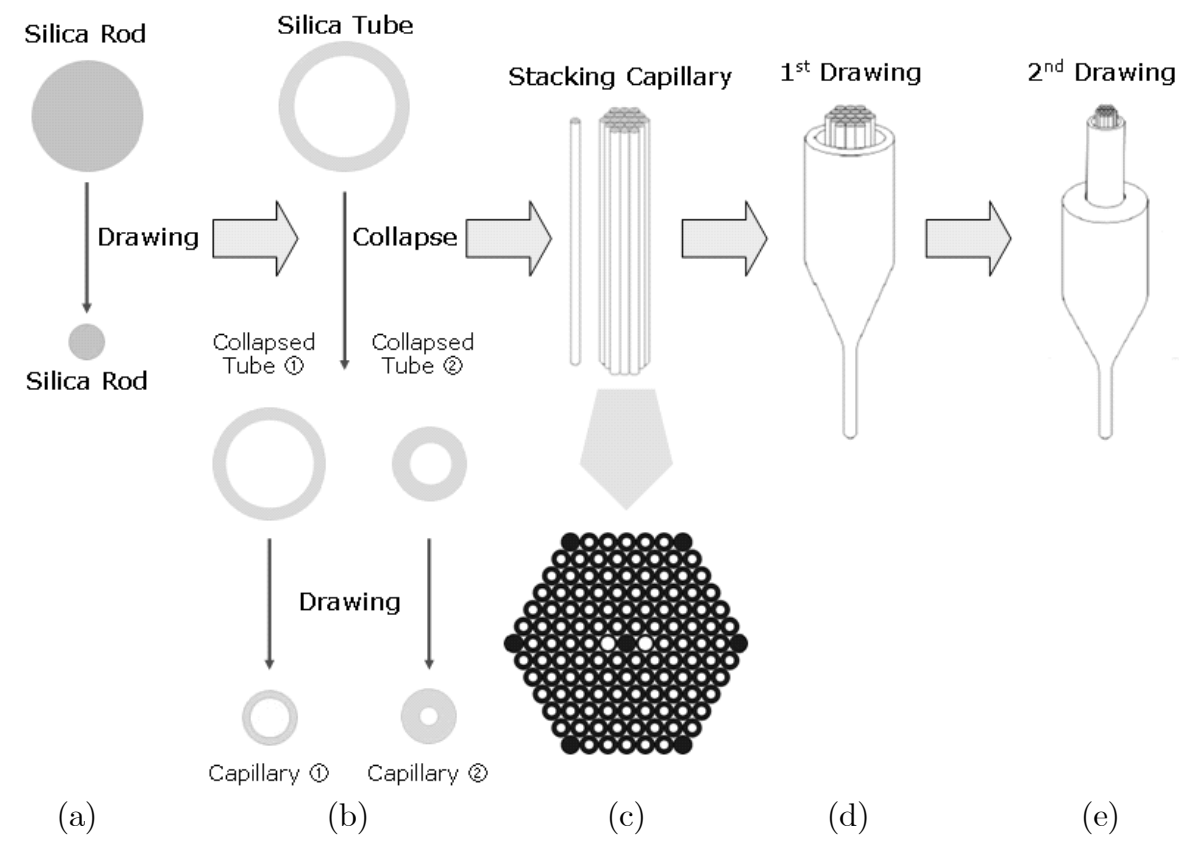

FIG. 2. Fabrication process of PM-PCFs; (a) drawing $1 \mathrm{~mm}$ silica rod for core, (b) collapsing two silica tubes to get the collapsed silica tubes with desired inner and outer diameter (Collapsed Tube (1) and Collapsed Tube (2), respectively) and drawing these collapsed tubes to $1 \mathrm{~mm}$ capillary (Capillary (1) and Capillary (2), respectively) (c) stacking capillaries based on hexagonal lattice, (d) the first drawing, and (e) the second drawing. 
$(1 \mathrm{~mm})$. Note that the small air hole to large air hole diameter ratio is determined in this collapsing process. The third process is to stack capillaries to obtain the fiber preform as shown in Fig. 2 (c). Before the stacking process, we completely sealed one end of each capillary by applying high temperature flame. After that, we stacked together end-sealed capillaries into a hexagonal structure. The fiber core was formed by replacing a center capillary with a $1 \mathrm{~mm}$ silica rod. For two large air holes, we replaced two capillaries near the core rod with larger inner hole capillaries. After then, the whole capillaries set was inserted into a jacket tube (out-jacket tube). The fourth process is the first drawing, in which the preform was drawn to the 'first drawn preform' having diameter of $5 \mathrm{~mm} \sim 6 \mathrm{~mm}$. Since there are micro defects between the stacked capillary and out-jacket tube, and these micro defects don't disappear at all when drawn to fiber, we constantly had to vacuum out air in the silica tube to eliminate these defects using the vacuum pump. Fig. 3 shows the schematic setup to control the internal pressure of silica tube.

Fig. 4 shows the cross section of stacked capillaries

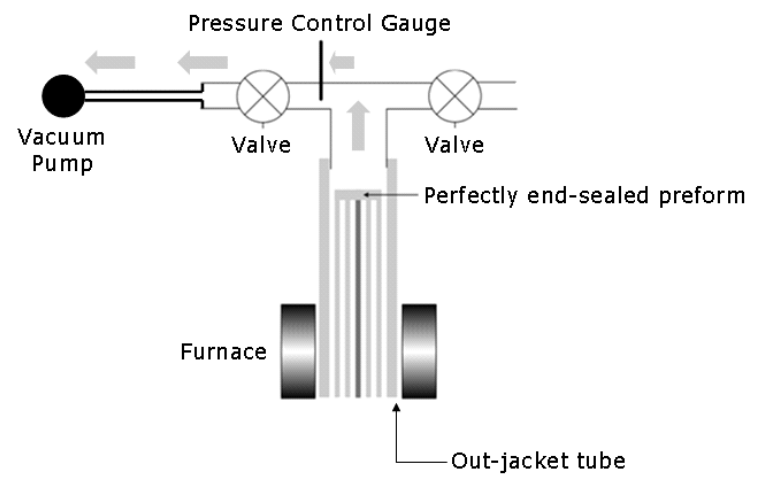

FIG. 3. Schematic setup to control the internal pressure of silica tube. Blue arrows indicate the flow of air in the silica tube.

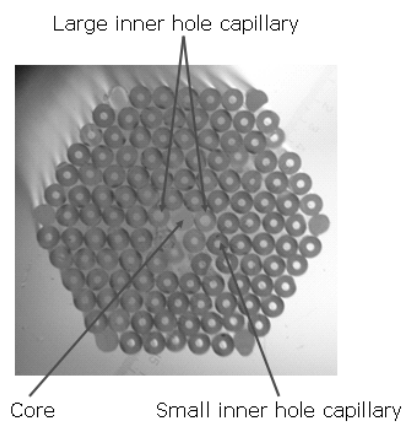

(a)

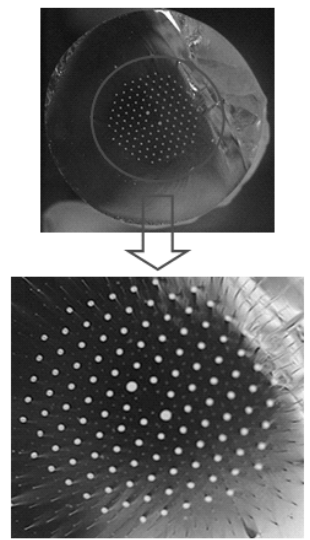

(b)

FIG. 4. Cross section of stacked capillaries (a) and the first drawn preform (b). and the first drawn preform. In order to prevent capillaries in the stacked capillaries from inflating too much or melting each other, we drew the first drawn preform with high tension and relatively low temperature (about $1900^{\circ} \mathrm{C}$ ) compared to a conventional drawing procedure. This is why so many interstitial holes among air holes in the first drawn preform exist. We could eliminate these interstitial holes during the second drawing process with reasonable temperature and pressure control.

The last fabrication process is to draw the fiber from the first drawn preform (the second drawing) using a conventional fiber drawing tower. In the initial stage of the second drawing process, a high temperature of about $2100^{\circ} \mathrm{C}$ is required to drop some end part of the preform below the heat-zone to start the process for coating and winding. The air holes in the fiber are melted together owing to the low tension and low viscosity at high temperature. The furnace temperature should be lowered to about $2000^{\circ} \mathrm{C}$ after the process for coating and winding started. In order to maintain uniform air hole structure, we had to control the furnace temperature, drawing speed, and tension loaded on the fiber. This is why high quality PCF is hard to fabricate. Fig. 5 shows the SEM (Scanning Electron Microscope) image of PM-PCF, and Table 1 summarizes

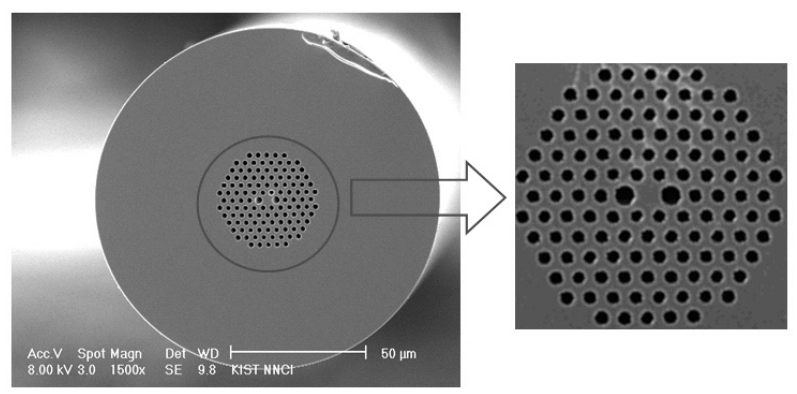

FIG. 5. SEM (Scanning Electron Microscope) image of PM-PCF.

TABLE 1. Physical properties of PM-PCF

\begin{tabular}{l|c}
\hline \hline \multicolumn{2}{c}{ Physical Property } \\
\hline Diameter of fiber $[\mu \mathrm{m}]$ & $125 \pm 3$ \\
\hline $\begin{array}{l}\text { Diameter of core } \\
(x \text { and } y \text { axis, respectively) }[\mu \mathrm{m}]\end{array}$ & $3.72 / 5.76$ \\
\hline Diameter of large air hole $\left(\mathrm{d}_{1}\right)[\mu \mathrm{m}]$ & 2.74 \\
\hline Diameter of small air hole $\left(\mathrm{d}_{2}\right)[\mu \mathrm{m}]$ & 1.61 \\
\hline $\begin{array}{l}\text { Ratio between small }\left(\mathrm{d}_{2}\right) \text { and } \\
\text { large air hole }\left(\mathrm{d}_{1}\right)\end{array}$ & 0.58 \\
\hline Hole-to-hole spacing $(\mathrm{pitch})(\Lambda)[\mu \mathrm{m}]$ & 3.41 \\
\hline Air filling fraction $(\mathrm{AFF})\left(\mathrm{d}_{2} / \Lambda\right)$ & 0.49 \\
\hline Diameter of holey region $[\mu \mathrm{m}]$ & 38.97 \\
\hline
\end{tabular}


physical properties of PM-PCF.

During the second drawing process, micro defects between the out-jacket silica tube and the first drawn preform may also appear. So we had to pull out air in silica tube constantly to eliminate these micro defects during the second drawing process. Fig. 6 shows the SEM image of PM-PCF that we didn't vacuum out air in silica tube in the second drawing. Here, the first drawn preform was same as the preform used at the PM-PCF in Fig. 5. In Fig. 6, there are many unintended and undesired air holes, which were formed from interstitial holes and micro defects between the first drawn preform and out-jacket silica tube since we didn't control the internal pressure of silica tube. In other words, our desired uniform air hole structure cannot be obtained without appropriate air pressure control.

\section{MEASUREMENT OF PM-PCF}

The basic optical properties of the PM-PCF such as normalized intensity, transmission loss, polarization dependent loss (PDL), birefringence, dispersion, and differential group delay (DGD) were measured.

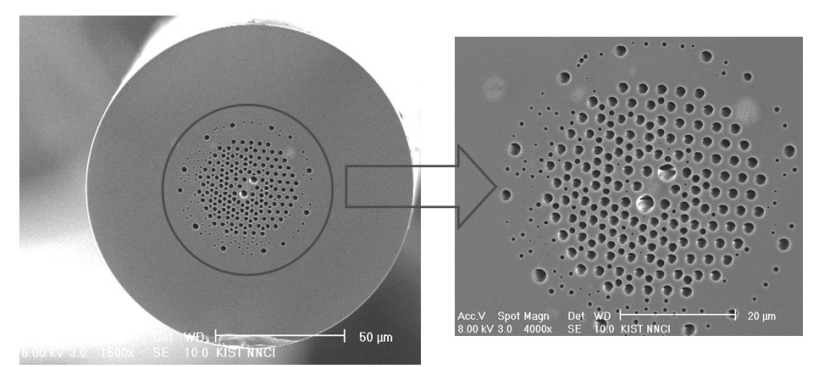

FIG. 6. SEM image of PM-PCF when we didn't vacuum out the air in the out-jacket tube during the second drawing process. The first drawn preform used here is same as that of PM-PCF in the Fig. 5.

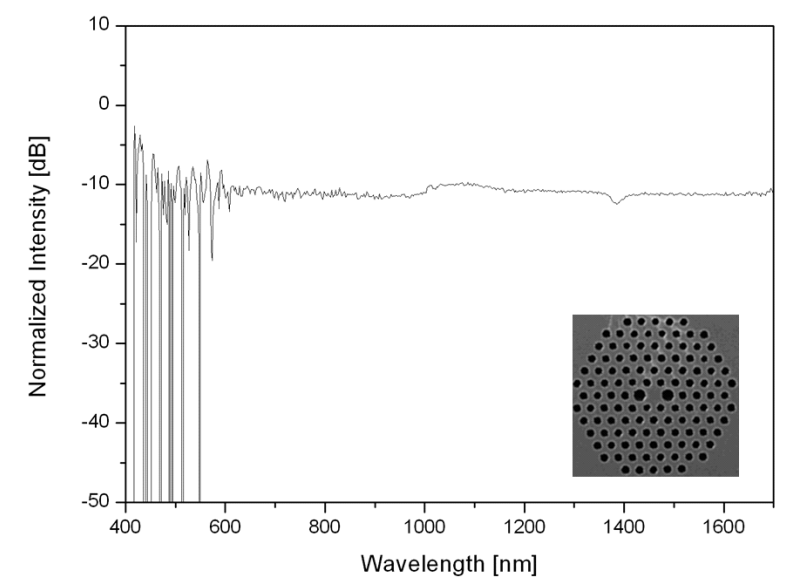

FIG. 7. Normalized intensity of PM-PCF. The inset is the cross section of the measured PM-PCF.
The normalized intensity is shown in Fig. 7 and the inset is the cross section of the measured PM-PCF. In order to measure the normalized intensity of the fabricated PM-PCF, we spliced two ends of $2 \mathrm{~m}$-long PM-PCF to two SMFs (Single Mode Fibers) and directly connected to an Optical Spectrum Analyzer (OSA, Ando AQ 6315B) and white light source (Ando AQ 4303B), respectively. From Fig. 7, the cut-off wavelength of PM-PCF was around $680 \mathrm{~nm}$ and we could see the $\mathrm{OH}^{-1}$ absorption around at $1390 \mathrm{~nm}$.

We measured the fiber transmission loss along the fiber length $(115 \mathrm{~m})$ using an optical time domain reflectometer (OTDR, Anritsu MW 9060A) at $1550 \mathrm{~nm}$, and the measured loss was $0.04 \mathrm{~dB} / \mathrm{m}$. Fig. 8 shows the OTDR waveform of PM-PCF.

The maximum loss difference between the two orthogonal polarization modes ( $x$ and $y$ axis, respectively) was measured from $1520 \mathrm{~nm}$ to $1620 \mathrm{~nm}$ by Tektronix PAT 9000B and Ando AQ4321D. First, we measured polarization dependent loss (PDL) of PANDA PM to compare with that of our PM-PCF. The length of fiber under test was $2 \mathrm{~m}$, and the PDL of the fibers at $1550 \mathrm{~nm}$ were $0.101 \mathrm{~dB}$ and $0.169 \mathrm{~dB}$, respectively. These results come from the average PDL of several times of measurements at $1550 \mathrm{~nm}$ for precision. It is clear from the above result that PDL of PM-PCF is somewhat larger than that of PANDA PM fiber and the two large air holes near core have little influence on PDL of PMPCF.

We also evaluated the birefringence of PM-PCF by building a Sagnac loop interferometer. [9] A Sagnac loop interferometer configuration was built using a standard $3 \mathrm{~dB}$ fiber coupler and $2 \mathrm{~m}$-long fiber under test (FUT). The two ends of $3 \mathrm{~dB}$ fiber coupler were fusion spliced to the FUT for making the Sagnac loop. The other two ends of the $3 \mathrm{~dB}$ coupler were directly connected to the light source (Ando AQ4321D) and an OSA (Ando AQ6315B), respectively. Measurements were performed by launching a linearly polarized light

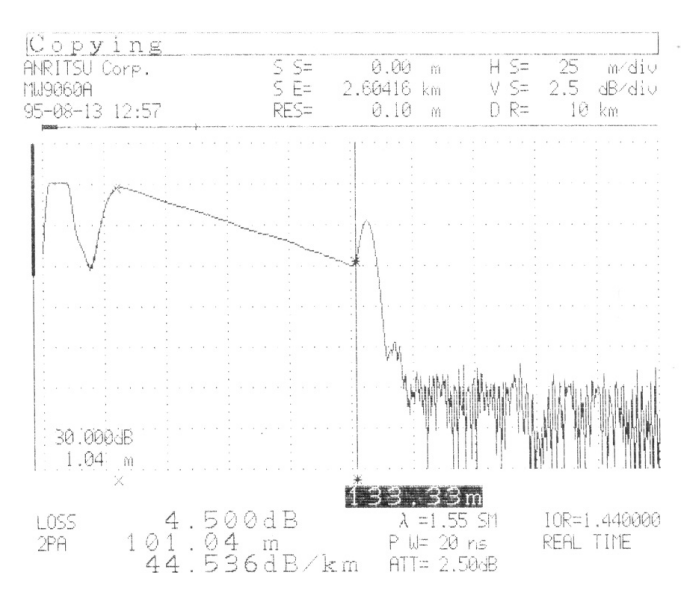

FIG. 8. OTDR waveform of PM-PCF. 


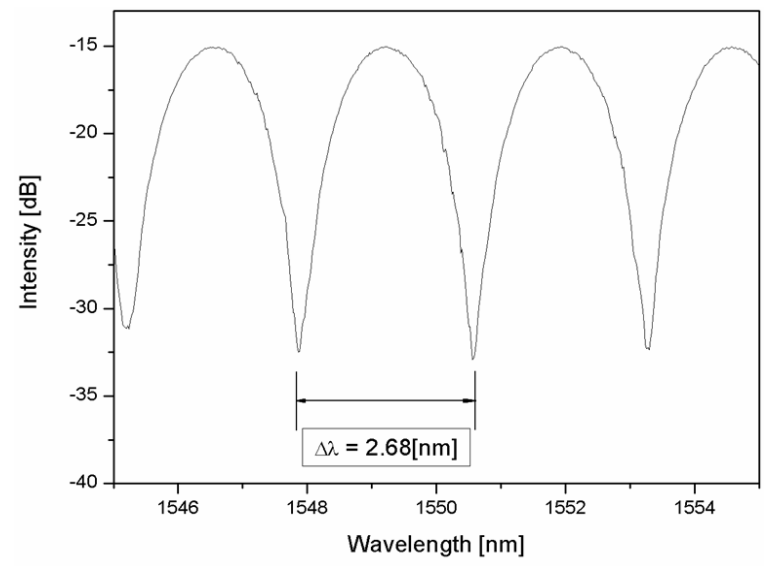

(a)

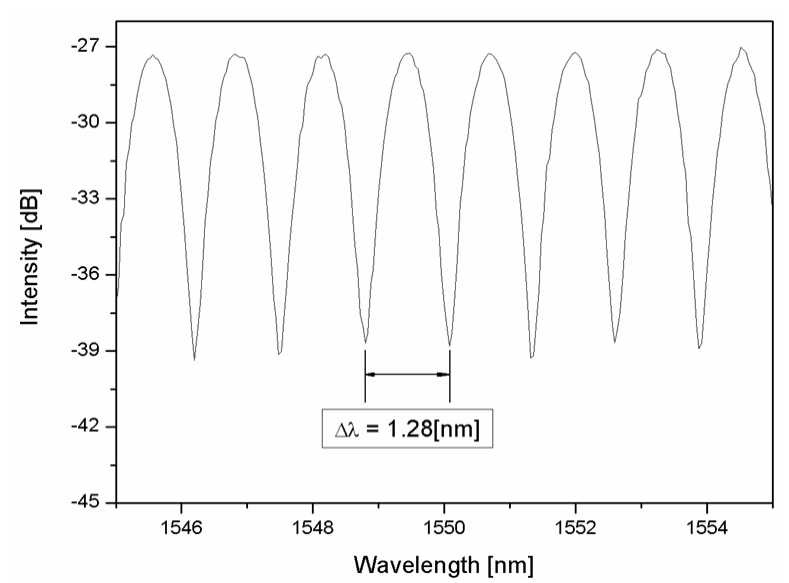

(b)

FIG. 9. Interference spectra of 2m-long PANDA PM fiber (a) and PM-PCF (b).

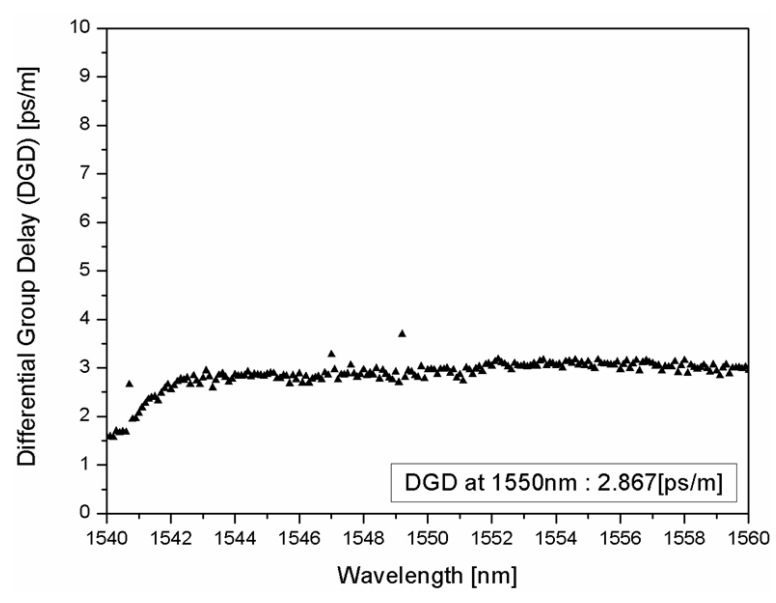

(a)

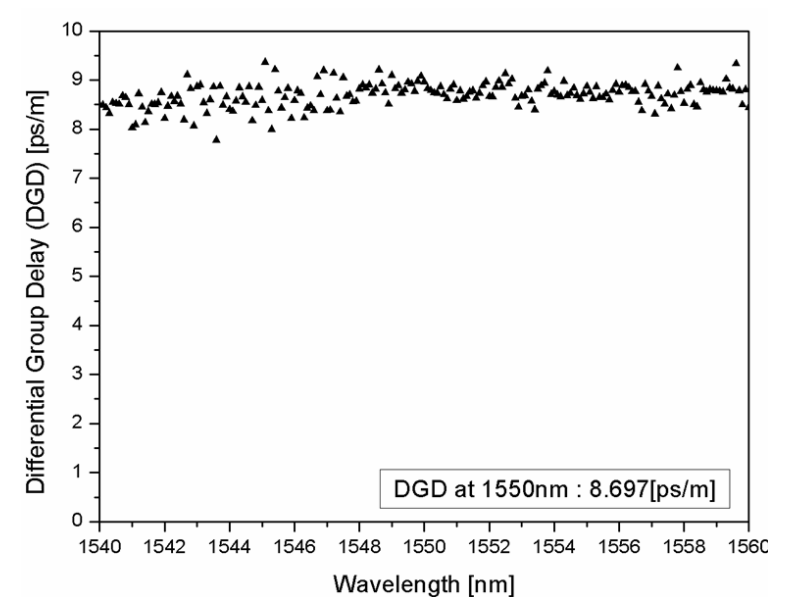

(b)

FIG. 10. Differential group delay (DGD) of PANDA PM fiber (a) and PM-PCF (b).

along the 2 m-long FUT. Fig. 9 shows the measured interference spectra of PANDA PM fiber and PM-PCF, respectively.

The relationship between the birefringence $(\Delta n)$ and wavelength spacing of the peaks $(\Delta \lambda)[9]$ is given by

$$
\Delta n=\frac{\lambda^{2}}{\Delta \lambda \cdot L}
$$

where $\lambda$ is operating wavelength and $L$ is the length of fiber.

The wavelength spacing of the two peaks was 2.68 $\mathrm{nm}$ at a wavelength of $1550 \mathrm{~nm}$ and the corresponding birefringence obtained from the above relation was $4.5 \times 10^{-4}$ for the PANDA PM fiber. For the PM-PCF, the wavelength spacing of the peaks was $1.28 \mathrm{~nm}$ and the corresponding birefringence was $1 \times 10^{-3}$. This result concludes that the birefringence of PM-PCF is of the order of $10^{-3}$, about three times larger than that of a conventional PANDA PM fiber.

Fig. 10 shows the differential group delay (DGD) wavelength dependence for PANDA PM fiber and PM-PCF, respectively. Measurements were performed using Tektronix PAT 9000B, which uses the JonesMatrix-Eigenanalysis (JME) method for the most complete characterization of the polarization mode dispersion (PMD). Since DGD is the difference in group delay at a specific wavelength between the fast ( $x$ axis) and slow (y axis) principal states of polarization, so we measured the dependency of the DGDs on wavelength. Fig. 10 shows the differential group delay (DGD) of PANDA $\mathrm{PM}$ fiber and PM-PCF, respectively.

We measured the dispersion characteristics of PMPCF using Advantest Optscope Q7750. The length of fiber under test was $115 \mathrm{~m}$, and the dispersion of $\mathrm{PM}-\mathrm{PCF}$ at $1550 \mathrm{~nm}$ was $56.3 \mathrm{ps} / \mathrm{nm} / \mathrm{km}$.

Table 2 compares the measured optical properties of fabricated PM-PCF and PANDA PM fiber. 
TABLE 2. Comparison of optical properties between PANDA PM fiber and PM-PCF

\begin{tabular}{l|c|c}
\hline \hline \multicolumn{1}{c|}{ Optical Property } & PANDA PM fiber & PM-PCF \\
\hline Polarization Dependent Loss (PDL) $[\mathrm{dB}]$ at $1550 \mathrm{~nm}$ & 0.101 & 0.169 \\
\hline Birefringence & $4.48 \times 10^{-4}$ & $1 \times 10^{-3}$ \\
\hline Differential Group Delay (DGD) $[\mathrm{ps} / \mathrm{m}]$ at $1550 \mathrm{~nm}$ & 2.867 & 8.697 \\
\hline
\end{tabular}

\section{CONCLUSION}

We fabricated PM-PCFs by using capillary stacking method. Since birefringence of PM-PCF is induced by the different effective index contrast between the two orthogonal axes, we stacked with one kind of capillaries based on hexagonal lattice and then replaced the two capillaries near core with other two capillaries having different inner diameter. The PM-PCFs had gone through the intermediate drawing process (the first drawing) with relatively low temperature and high tension in order not to make the preform stay in the furnace for a long time. This prevented the air holes in the preform from melting together and inflating too much. The various optical properties such as normalized intensity, transmission loss, PDL, birefringence, dispersion and DGD were measured, and compared with those of conventional PANDA PM fiber.

\section{ACKNOWLEDGMENT}

This work was partially supported by the IT R\&D program of MIC/IITA [2005-S099-03, Development of photonic crystal optical fibers and their applications technology for high speed optical communication system].

${ }^{*}$ Corresponding author: sblee@kist.re.kr

\section{REFERENCES}

[1] T. A. Burks, J. C. Knight, and P. St. J. Russell, "Endlessly single-mode photonic crystal fiber", Opt. Lett., vol. 22, no. 13, pp. 961-963, 1997.

[2] T. M. Monro, D. J. Richarson, N. G. R. Broderick, and P. J. Bennett, "Holey optical fibers: An efficient modal model," IEEE J. Lightwave Technol., vol. 17, no. 6, pp. 1093-1102, 1999.

[3] T. A. Birks, D. Mogilevtsev, J. C. Knight, and P. St. J. Russell, "Dispersion compensation using single-material fibers," IEEE Photonics Technol. Lett., vol. 11, no. 6, pp. 674-676, 1999.

[4] A. Ortigosa-Blanch, J. C. Knight, W. J. Wadsworth, J. Arriaga, B. J. Mangan, T. A. Burks, and P. St. J. Russell, "Highly birefringent photonic crystal fibers", Opt. Lett., vol. 25, no. 18, pp. 1325-1327, 2000.

[5] N. G. R. Broderick, T. M. Monro, P. J. Bennett, and D. J. Richardson, "Nonlinearity in holey optical fibers: Measurement and future opportunities”, Opt. Lett., vol. 24, no. 20, pp. 1084-1100, 2000.

[6] T. Sorensen, J. Breong, A. Bjarklev, E. Kundsen, and E. E. Barkou Libori, "Macro-bending loss properties of photonic crystal fibre”, Electron. Lett., vol. 37, no. 5, pp. 287-288, 2001.

[7] T. Hosaka, K. Okamoto, T. Miya, Y. Sasaki, and T. Edahiro, "Low-loss single polarisation fibres with asymmetrical strain birefringence", Electron. Lett., vol. 17, no. 15, pp. 530-531, 1981.

[8] R. D. Birch, D. N. Payne, and M. P. Varnham, "Fabrication of polarisation-maintaining fibres using gas-phase etching", Electron. Lett., vol. 18, no. 24, pp. 1036-1038, 1982.

[9] Do-Hyun Kim and Jin U. Kang, "Sagnac loop interferometer based on polarization maintaining photonic crystal fiber with reduced temperature sensitivity", Opt. Express, vol. 12, no. 19, pp. 4490-4495, 2004. 